\title{
3CAP: Categorizing the Cognitive Capabilities of Alzheimer's Patients in a Smart Home Environment
}

\author{
Kate M. Bowers \\ Oakland University \\ Rochester, MI, USA \\ kmlabell@oakland.edu
}

\author{
Reihaneh H. Hariri \\ Oakland University \\ Rochester, MI, USA \\ rhosseinzadehha@oakland.edu
}

\author{
Katey A. Price \\ Albion College \\ Albion, MI, USA \\ kprice@albion.edu
}

\begin{abstract}
Alzheimer's disease is a progressive illness that affects more than 5.5 million people in the United States with no effective cure or treatment. Symptoms of the disease include declines in memory and speech abilities and increases in aggression and insomnia. Recent research suggests that NLP techniques can detect early cognitive decline as well as monitor the rate of decline over time. The processed data can be used in a smart home environment to enhance the level of home care for Alzheimer's patients. This paper proposes early-stage research in software engineering and natural language processing for quantifying and evaluating the patient's cognitive state to determine the required level of support in a smart home.
\end{abstract}

\section{CCS CONCEPTS}

- Human-centered computing $\rightarrow$ Natural language interfaces; - Social and professional topics $\rightarrow$ Medical records; Software selection and adaptation; Seniors; - Computing methodologies $\rightarrow$ Information extraction;

\section{KEYWORDS}

natural language processing, smart home, self-adaptive systems, Alzheimer's disease, cyber-physical systems

\section{ACM Reference Format:}

Kate M. Bowers, Reihaneh H. Hariri, and Katey A. Price. 2018. 3CAP: Categorizing the Cognitive Capabilities of Alzheimer's Patients in a Smart Home Environment. In Proceedings of the 4th ACM SIGSOFT International Workshop on NLP for Software Engineering (NL4SE '18), November 4, 2018, Lake Buena Vista, FL, USA. ACM, New York, NY, USA, 4 pages. https: //doi.org/10.1145/3283812.3283824

\section{INTRODUCTION}

Over 5.5 million people in the United States have been diagnosed with Alzheimer's disease [1], making the disease a top-ten leading cause of death with no cure or effective form of treatment. As a result, 15 million family members and friends provide unpaid care to those suffering from Alzheimer's. In the next 11 years the elderly population (i.e., ages 65 and older) will grow by $60 \%$ while younger populations remain the same $[3,19]$. The negative effects

Permission to make digital or hard copies of all or part of this work for personal or classroom use is granted without fee provided that copies are not made or distributed for profit or commercial advantage and that copies bear this notice and the full citation on the first page. Copyrights for components of this work owned by others than ACM must be honored. Abstracting with credit is permitted. To copy otherwise, or republish, to post on servers or to redistribute to lists, requires prior specific permission and/or a fee. Request permissions from permissions@acm.org.

NL4SE '18, November 4, 2018, Lake Buena Vista, FL, USA

(C) 2018 Association for Computing Machinery.

ACM ISBN 978-1-4503-6055-5/18/11 . \$15.00

https://doi.org/10.1145/3283812.3283824 of moving to a dementia unit at a nursing care facility (e.g., stress, costs, cognitive decline) can be delayed if the individual is allowed to stay at home for as long as possible [24]. Research suggests that installing a variety of embedded devices that monitor patient health and safety in the home can delay institutionalization [11]. Establishing a medically-oriented smart home for early-stage Alzheimer's patients may help combat the impending manpower deficit, provide a cost-effective solution for patients, and reduce the demand on family or friends to become full-time caregivers.

CAL (Cognitive Assisted Living), an architecture for a self-adaptive smart home system, was recently introduced to support Alzheimer's patients by changing its configurations at run time in response to the decline in the patient's cognitive health [11]. Configuration changes may include overriding patient decisions in the household if the decisions put the patient in a potentially dangerous state (e.g., if the patient releases gas in a fireplace but forgets to ignite it). To accurately determine changes in configurations and the patient's cognitive capabilities, CAL interacts with the patient on a semi-regular basis (e.g., every day or week). Currently, patients are diagnosed with Alzheimer's disease after a doctor asks the patient a series of questions designed to test the patient's verbal, spatial, and reasoning skills $[2,4,18]$. However, a single evaluation may not provide enough data for an adaptive smart home system to determine an optimal reconfiguration strategy. For example, a patient in the early stages of Alzheimer's may not need as high of a degree of monitoring or as many decision overrides as a patient in the moderate stages of the disease. To this end, we introduce 3CAP (Categorizing the Cognitive Capabilities of Alzheimer's Patients) that combines natural language processing (NLP) and software engineering techniques to evaluate the decline in verbal and reasoning skills associated with Alzheimer's disease and provide as input the level of support required to a medically-oriented smart home.

3CAP presents early-stage research to implement the initial steps of CAL with regard to interacting with patients and evaluating the required level of support in the smart home. CAL provides the interface to the patient (e.g., through a tablet) as well as a predetermined set of questions similar to memory screening questions. 3CAP accepts from CAL an audio file of patient responses, converts the audio file to a string of text, uses NLP data to evaluate how well the patient answered the questions, and finally sends CAL a variable indicating how much support to provide the patient. Although 3CAP evaluates the patient responses, CAL is ultimately responsible for determining optimal reconfiguration strategies and when or whether to perform reconfigurations. For example, if 3CAP determines that a patient consistently answers questions correctly, CAL may turn off sensors that monitor sleep and activity, allow manual changes to the thermostat or home security system, and 
disable reminders to take medications. In contrast, if 3CAP finds that the patient begins to consistently answer questions incorrectly, CAL may perform a reconfiguration to turn on sleep and activity sensors, automatically operate the thermostat and security system, and enable prescription reminders.

3CAP evaluates a patient's response to a mental health screening question in five parts. First, the patient's recorded response is converted from an audio file to a string of text. Second, NLP techniques such as latent semantic analysis (LSA), part of speech (POS) tagging, and term frequency-inverse document frequency (TF-IDF) evaluate sentence length, sentence validity, word complexity, and word validity, while N-gram modeling is used to count the number of syllables per word [6, 12, 20,23,25]. Third, a set of utility functions $[10,21,26]$ are defined to quantify the patient's response with regard to syntax, vocabulary, and accuracy metrics. Fourth, a linear weighted sum provides an overall score to the question, where a weight is assigned to each utility function to indicate the relative importance of each metric in evaluating the question. Finally, the response score is categorized into (1) highly independent, (2) somewhat independent, (3) somewhat dependent, and (4) highly dependent and sent to CAL.

The remainder of the paper is organized as follows. Section 2 presents background information on Alzheimer's disease, smart homes, and utility functions in self-adaptive systems. Section 3 outlines our approach and Section 4 details related work. Finally, Section 5 provides a discussion and presents future applications.

\section{BACKGROUND}

This section provides background information on Alzheimer's disease, smart homes, and utility functions in goal modeling.

\subsection{Early-Stage Alzheimer's Disease}

Alzheimer's disease, the most common of over two dozen types of dementia, is caused by a buildup of plaques and proteins in the brain, causing cell death and brain shrinkage over time [1]. Patients afflicted with Alzheimer's experience behavioral changes (e.g., generally becoming more aggressive), loss in spatial and reasoning skills (e.g., have trouble budgeting, organizing, or driving), and progressive aphasia (i.e., the gradual loss of speech abilities) [1] Although patients in the early stages of Alzheimer's may be able to function independently, the progressive nature of the illness will eventually require patients to have assistance with activities of daily living (ADLs) such as bathing, eating, getting dressed, or using the bathroom $[1,8]$.

\subsection{Smart Homes}

Smart homes are generally realized as Internet of Things (IoT) ecosystems and can provide users extended services not normally included in a typical household [14]. Extensive research in the smart home domain includes health care applications for elderly residents $[9,14,15]$. Smart homes can provide care to patients without inducing the confusion and stress associated with transfers to nursing homes or extended care facilities [24]. Some examples of medical smart home devices may include wearables (e.g., smart watches, pedometers), door sensors for potential wander risks, thermostats (e.g., Nest), monitoring prescription consumption, and voice-activated capabilities such as Amazon's Alexa and Google Home [15, 27]. These smart home devices typically have static configurations that may become sub-optimal over the patient's lifetime (i.e., changing medication or activity levels).

\subsection{Utility Functions}

Utility functions have been used to quantify the degree of satisfaction (i.e., satisficement) of software requirements at design time and run time $[10,21,26]$. A utility value of 0.0 equates to a requirement violation, 1.0 equates to complete requirement satisfaction, and any value within range of $(0.0,1.0)$ indicates a degree of requirement satisficement [7].

For example, a smart home may have a requirement to minimize the number of inactive devices, where a device may be considered inactive if it cannot send or receive data. Expression 1 shows the utility function for the requirement to Achieve [Num Inactive Devices $==0]$.

$$
\text { util }= \begin{cases}1.0 & \text { if Num Inactive Devices }==0 \\ x & \text { if } 0<\text { Num Inactive Devices }<20 \% \text { of total devices } \\ 0.0 & \text { if Num Inactive Devices } \geq 20 \% \text { of total devices }\end{cases}
$$

If no devices are inactive (i.e., all devices are sending and receiving data), then the utility value is 1.0 . If at least one device, but less than $20 \%$ of the total number of devices in the smart home, are inactive, the utility value linearly decreases (e.g., if $10 \%$ of the devices are inactive, then the utility value is 0.5 ). If more than $20 \%$ of the devices in the smart home are inactive, the utility value is 0.0 to indicate a requirement violation.

\section{APPROACH}

This section overviews the 3CAP technique. Figure 1 presents a data flow diagram that illustrates the process used by 3CAP, taking as input an audio file that records patient responses and outputs a variable with values ranging from 1 to 4 . An output value of 1 indicates the lowest level of required support and a value of 4 indicates the highest level of required support. We next detail each step of the 3CAP technique.

(1) Convert Speech to Text. Questions 2-6 demonstrate examples of questions performed in a doctor's office during a cognition and memory screening test $[2,4,18]$.

\begin{tabular}{|l|}
\hline What is today's date? \\
\hline $\begin{array}{l}\text { Tell me as many animals as you can think of in } 30 \\
\text { seconds. }\end{array}$ \\
\hline $\begin{array}{l}\text { Can you tell me something that happened in the } \\
\text { news recently? }\end{array}$ \\
\hline $\begin{array}{l}\text { Remember these 3-5 words and repeat them back to } \\
\text { me. }\end{array}$ \\
\hline \hline Spell a word (e.g., WORLD) backwards. \\
\hline
\end{tabular}

Note that each question can be varied slightly but still generate similar patient responses. For example, Question 3 may replace the word "animals" with other categories such as fruit, vegetables, plants, etc. while Questions 5 and 6 can list any number of randomly generated words from a predetermined database.

To perform NLP, the audio file must first be converted to a string of text. A variety of external services offer state-of-the-art speech 




Figure 1: Data flow diagram of 3CAP technique

conversion capabilities, such as Google Cloud Speech API or Amazon Alexa Voice, and are applied here.

(2) Apply NLP to Audio File. Traditionally, memory screening questions evaluate patient responses predominantly in terms of accuracy (i.e., whether the patient answered each question correctly or incorrectly) $[2,4,18]$. However, poor sentence structure, vocabulary, and semantic intent could additionally be potential indicators of cognitive decline in Alzheimer's patients [20,25]. Similarly, we consider three verbal classifications as software requirements: (1) syntax, (2) vocabulary, and (3) accuracy. Each question is divided into these three classifications (i.e., Question 1 is evaluated with syntax, vocabulary, and accuracy). Failures in these requirements may be used indicate the decline in verbal and reasoning skills of Alzheimer's patients.

3CAP uses POS tagging, LSA, and TF-IDF to evaluate sentence length, sentence validity, word complexity, and word validity [6, $12,20,23,25]$. These four metrics are further used to evaluate the syntax and accuracy of the patient's response to a screening question. Similarly, the syllable-N-gram model is used to count the number of syllables per word that is then applied in the evaluation of the vocabulary in the patient's response.

(3) Calculate Utility Functions. The syntax, vocabulary, and accuracy metrics are modeled as software requirements in a selfadaptive system and evaluated using utility functions [10, 21, 26]. For example, to evaluate the accuracy of Question 1, Equation 7 indicates that the patient completely satisfied the question, with a utility value of 1.0 , if the correct date, month, and year were given. If all three fields are incorrect, the patient completely failed, yielding a utility value of 0.0 . However, if the patient responds with a mixture of right and wrong answers, the utility function is a linear value $x$ between the upper and lower thresholds For example, Question 1's accuracy is 0.67 if the patient correctly gave the month and year but the wrong date.

$$
\text { util( } \text { accuracy })= \begin{cases}1.0 & \text { if date, month, and year are correct } \\ x & \text { if } 0<\text { Num Correct }<\text { All Correct } \\ 0.0 & \text { if date, month, and year are incorrect }\end{cases}
$$

Additional utility functions evaluate the vocabulary and syntax of Question 1. Note that utility functions for the same verbal categories will vary for different questions. For example, the accuracy utility value of Question 2 may be 1.0 if the patient can list more than five items in the given category in contrast to Question 1, where the accurate requirement is only completely satisfied if the patient gets all three facts correct.

(4) Calculate Score. Once utility values for each verbal classification are determined, a linear weighted sum evaluates the satisficement of the entire question, as a single question does not necessarily use each verbal category equally. For example, Questions 2, 5, and 6 focus primarily on accuracy to test patient recall and do not ask the patient to construct sentences. In contrast, Question 4 allows the patient to tell a story or one-sided conversation that uses full sentences and can therefore be analyzed syntactically. Furthermore, Question 3 tests the patient's vocabulary by asking for a list of as many words as possible in a given category, but also analyzes accuracy as objects should not be listed with animals. Therefore, we propose to apply weights to the syntax, vocabulary, and accuracy for each question. The patient's response to a single screening question is evaluated as an aggregate utility function in Equation 8.

$$
\begin{aligned}
& u t i l_{-} v a l u e=\left(u t i l_{\text {accuracy }} * w_{\text {eight }} \text { accuracy }\right)+ \\
& \left(\text { util }_{\text {vocabulary }} * \text { weight }_{\text {vocabulary }}\right)+ \\
& \text { (util } \left.l_{\text {syntax }} * \text { weight }_{\text {syntax }}\right)
\end{aligned}
$$

Note that the summation of the verbal category weights must equal 1.0. Each weight associated with a verbal category varies per question. The summation of weighted utility values is output as a response score.

(5) Categorize Support. A response score estimates the patient's cognitive capabilities that can be used to classify the level of support a smart home needs to provide, shown in Table 1.

Table 1: Categories of required level of support based on patient response score.

\begin{tabular}{|l|c|}
\hline Level of Required Support & Response Score \\
\hline (1) Highly independent & $0.75<\mathrm{x}<=1.0$ \\
\hline (2) Somewhat independent & $0.5<\mathrm{x}<=0.75$ \\
\hline (3) Somewhat dependent & $0.25<\mathrm{x}<=0.5$ \\
\hline (4) Highly dependent & $0.0<=\mathrm{x}<=0.25$ \\
\hline
\end{tabular}

The response score, with values ranging from 1.0 to 0.0 , is linearly divided into four categories. A response that answers the given question perfectly has an associated response score of 1.0, resulting in a highly independent state for the smart home (e.g., lower degree of health monitoring and medication reminders). A completely incorrect response is associated with a response score of 0.0 and puts the smart home in a highly dependent state (e.g., the highest degree of health monitoring). The categories of somewhat independent and somewhat dependent correspond to moderate degrees of health monitoring (e.g., enable sensors in the household to collect data but do not override manual thermostat settings). 


\section{RELATED WORK}

NLP is increasingly being used in medical applications, such as translating doctor notes or medical reports [5, 16, 22]. Mobile apps [13,29] and digital tests [17, 28, 30] have allowed patients to take screening evaluations from the comfort of their homes and obtain a diagnosis. Other approaches similar to 3CAP use NLP to detect or predict Alzheimer's disease in patients [6, 20, 23] but do not extend their evaluations to smart homes. Although certain smart homes address a variety of health care needs, including healthy, autistic, or elderly users [9, 14], the progressive nature of Alzheimer's disease may be better addressed with an adaptive smart home [11].

\section{DISCUSSION}

This paper presented 3CAP, an early-stage approach that combines NLP, utility functions, and linear weighted sums to evaluate verbal responses of Alzheimer's patients to memory screening questions and determine the required level of support for a medically-oriented smart home. 3CAP first converts the input audio file to text and uses NLP techniques to extract data from the recorded patient response. The NLP data is used to calculate utility functions that evaluate the syntax, accuracy, and vocabulary. A linear weighted sum applies weights to each utility function according to the intent of the question. Finally, the linear weighted sum is used as a response score to categorize the required level of support, where a value of 1 indicates the lowest level of system support and 4 indicates the highest level of system support. The smart home then determines an appropriate response (i.e., increase or decrease the degree of monitoring patient activities and health).

Future directions for this research include simulating 3CAP against e-books for specific age groups, implementing the technique via an Android tablet, obtaining user stories to improve patient feedback while avoiding confusion, and building the smart home as a whole. Furthermore, obtaining data from actual patients rather than simulated input may yield more accurate results and unveil patterns not yet discovered in the decline of speaking abilities associated with Alzheimer's disease, especially if the processed data can be validated with a doctor's diagnosis.

\section{ACKNOWLEDGEMENTS}

This work has been supported in part by NSF grant CNS-1657061, the Michigan Space Grant Consortium, the Comcast Innovation Fund, and Oakland University. Any opinions, conclusions, or recommendations expressed in this material are those of the author(s).

\section{REFERENCES}

[1] Alzheimer's Association. 2017. 2017 Alzheimer's disease facts and figures Alzheimer's \& Dementia 13, 4 (2017), 325-373.

[2] H. Brodaty, N. Kemp, and L. Low. 2004. Characteristics of the GPCOG, a screening tool for cognitive impairment. International journal of geriatric psychiatry 19, 9 (2004), 870-874.

[3] U.S. Census Bureau. 2017. The Nation's Older Population Is Still Growing, Census Bureau Reports. (2017). https://www.census.gov/newsroom/press-releases/2017/ cb17-100.html

[4] H. Buschke, G. Kuslansky, M. Katz, W. Stewart, M. Sliwinski, and R. Eckholdt, H.and Lipton. 1999. Screening for dementia with the memory impairment screen. Neurology 52, 2 (1999), 231-231.

[5] T. Cai, A. Giannopoulos, S. Yu, T. Kelil, B. Ripley, K. Kumamaru, F. Rybicki, and D. Mitsouras. 2016. Natural language processing technologies in radiology research and clinical applications. Radiographics 36, 1 (2016), 176-191.
[6] P. Chen, H. Zafar, M. Galperin-Aizenberg, and T. Cook. 2017. Integrating natural language processing and machine learning algorithms to categorize oncologic response in radiology reports. fournal of digital imaging (2017), 1-7.

[7] L. Chung, B. Nixon, E. Yu, and J. Mylopoulos. 2000. Non-functional Requirements. Software Engineering (2000).

[8] Mayo Clinic. 2015. Alzheimer's disease: How the disease progresses. (2015). http://www.mayoclinic.org/diseases-conditions/alzheimers-disease/ in-depth/alzheimers-stages/art-20048448

[9] R. Costa, D. Carneiro, P. Novais, L. Lima, J. Machado, A. Marques, and J. Neves. 2009. Ambient Assisted Living. In 3rd Symposium of Ubiquitous Computing and Ambient Intelligence 2008. 86-94.

[10] P. deGrandis and G. Valetto. 2009. Elicitation and Utilization of Applicationlevel Utility Functions. In Proc. of the 6th International Conference on Autonomic Computing (ICAC '09). ACM, 107-116.

[11] E. Fredericks, K. Bowers, K. Price, and R. Hariri. 2018. CAL: A Smart Home Environment for Monitoring Cognitive Decline. In 2018 IEEE 38th International Conference on Distributed Computing Systems (ICDCS). IEEE.

[12] V. Gupta and G. Lehal. 2010. A survey of text summarization extractive techniques. fournal of emerging technologies in web intelligence 2, 3 (2010), 258-268.

[13] Y. Huang, A. Singh, S. Chen, F. Sun, C. Huang, and S. Liu. 2017. Validity of a Novel Touch Screen Tablet-Based Assessment for Mild Cognitive Impairment and Probable AD in Older Adults. Assessment (2017), 1073191117748395.

[14] J. Kientz, S. Patel, B. Jones, E. Price, E. Mynatt, and G. Abowd. 2008. The Georgia Tech Aware Home. In CHI'08 Extended Abstracts on Human Factors in Computing Systems. $3675-3680$.

[15] T. Kleinberger, M. Becker, E. Ras, A. Holzinger, and P. Müller. 2007. Ambient Intelligence in Assisted Living: Enable Elderly People to Handle Future Interfaces. In Universal Access in Human-Computer Interaction. Ambient Interaction. Springer, Berlin, Heidelberg, 103-112.

[16] P. Lakhani, W. Kim, and C. Langlotz. 2012. Automated extraction of critical test values and communications from unstructured radiology reports: an analysis of 9.3 million reports from 1990 to 2011. Radiology 265, 3 (2012), 809-818.

[17] D Levinson, D Reeves, J Watson, and M Harrison. 2005. Automated neuropsychological assessment metrics (ANAM) measures of cognitive effects of Alzheimer's disease. Archives of Clinical Neuropsychology 20, 3 (2005), 403-408.

[18] M. Mendiondo, J. Ashford, R. Kryscio, and F. Schmitt. 2003. Designing a brief alzheimer screen (BAS). Journal of Alzheimer's Disease 5, 5 (2003), 391-398.

[19] World Health Organization. 2015. World report on ageing and health. World Health Organization.

[20] S. Orimaye, J. Wong, K. Golden, C. Wong, and I. Soyiri. 2017. Predicting probable AlzheimerâĂŹs disease using linguistic deficits and biomarkers. BMC bioinformatics 18, 34 (2017), 1-13.

[21] A. Ramirez and B. Cheng. 2011. Automatically Deriving Utility Functions for Monitoring Software Requirements. In Proceedings of the 2011 International Conference on Model Driven Engineering Languages and Systems Conference. Wellington, New Zealand, 501-516.

[22] L. Schwartz, D. Panicek, A. Berk, Y. Li, and H. Hricak. 2011. Improving communication of diagnostic radiology findings through structured reporting. Radiology 260, 1 (2011), 174-181.

[23] D. Shibata, S. Wakamiya, A. Kinoshita, and E. Aramaki. 2016. Detecting Japanese patients with AlzheimerâÁŹs disease based on word category frequencies. In Proceedings of the Clinical Natural Language Processing Workshop (ClinicalNLP). $78-85$.

[24] M. Skubic, G. Alexander, M. Popescu, M. Rantz, and J. Keller. 2009. A smart home application to eldercare: Current status and lessons learned. Technology and Health Care 17, 3 (2009), 183-201.

[25] C. Thomas, V. Keselj, N. Cercone, K. Rockwood, and E. Asp. 2005. Automatic detection and rating of dementia of Alzheimer type through lexical analysis of spontaneous speech. In Mechatronics and Automation, 2005 IEEE International Conference, Vol. 3. IEEE, 1569-1574.

[26] W. Walsh, G. Tesauro, J. Kephart, and R. Das. 2004. Utility functions in autonomic systems. In Proceedings of the First IEEE International Conference on Autonomic Computing. IEEE Computer Society, 70-77.

[27] J. Whittle, P. Sawyer, N. Bencomo, B. H. C. Cheng, and J.M. Bruel. 2009. RELAX: Incorporating Uncertainty into the Specification of Self-Adaptive Systems. In 17th IEEE International Requirements Engineering Conference (RE'09). 79-88.

[28] K. Wild, D. Howieson, F. Webbe, A. Seelye, and J. Kaye. 2008. Status of computerized cognitive testing in aging: a systematic review. Alzheimer's \& dementia: the journal of the Alzheimer's Association 4, 6 (2008), 428-437.

[29] G. Zorluoglu, M. Kamasak, L. Tavacioglu, and P. Ozanar. 2015. A mobile application for cognitive screening of dementia. Computer methods and programs in biomedicine 118, 2 (2015), 252-262.

[30] S. Zygouris and M. Tsolaki. 2015. Computerized cognitive testing for older adults: a review. American fournal of Alzheimer's Disease \& Other Dementias ${ }^{\circledR} 30,1$ (2015), 13-28. 